Supporting information

\title{
UV-Filtering Cellulose Nanocrystal/Carbon Quantum Dot Composite Films for Light Conversion in Glass Windows
}

Jian Gan ${ }^{1,3}$, Yan $W^{1,3^{*}}$, Feng Yang ${ }^{2}$, Xinyu $W u^{1,3}$, Yajing, Wang ${ }^{1,3}$, Jing Wang ${ }^{1,3}$.

1. College of Furnishings and Industrial Design, Nanjing Forestry University, Nanjing 210037, China

2. Fashion Accessory Art and Engineering College, Beijing Institute of Fashion Technology, Beijing 100029, China

3. Co-Innovation Center of Efficient Processing and Utilization of Forest Resources, Nanjing Forestry University, Nanjing 210037, China.

*Corresponding author: Yan Wu, E-mail: wuyan@njfu.edu.cn 


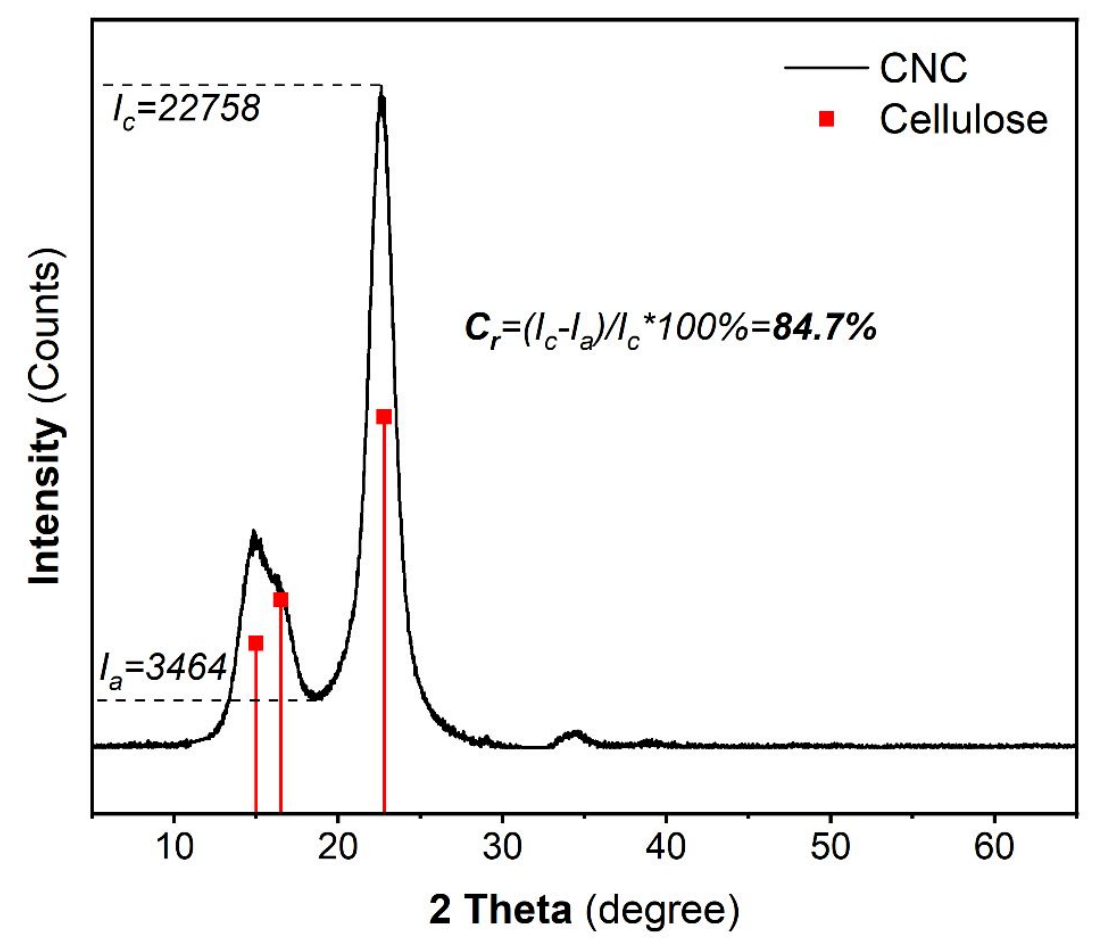

Figure s1. XRD diagram of the nanocellulose crystals (CNC) 


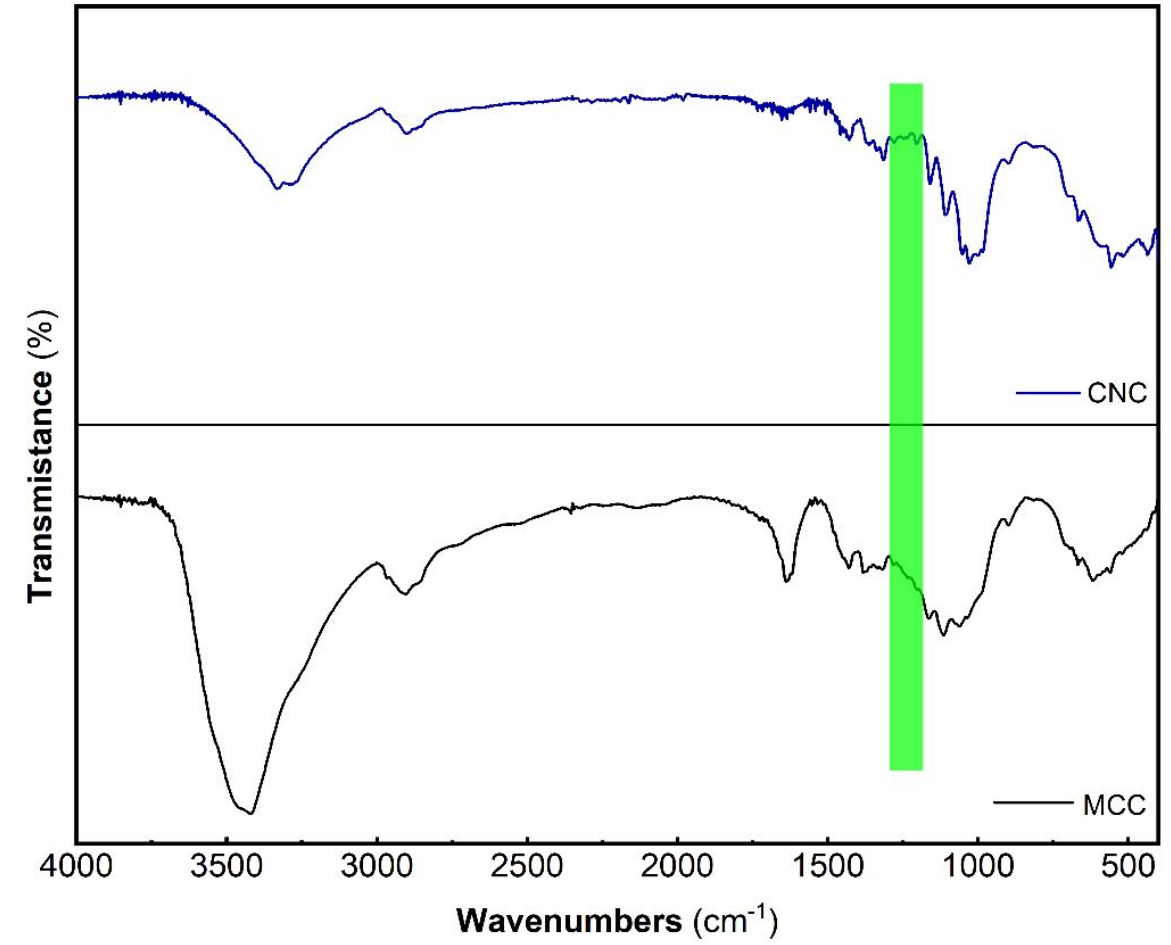

Figure s2. FTIR spectrum of nanocellulose crystals (CNC) 

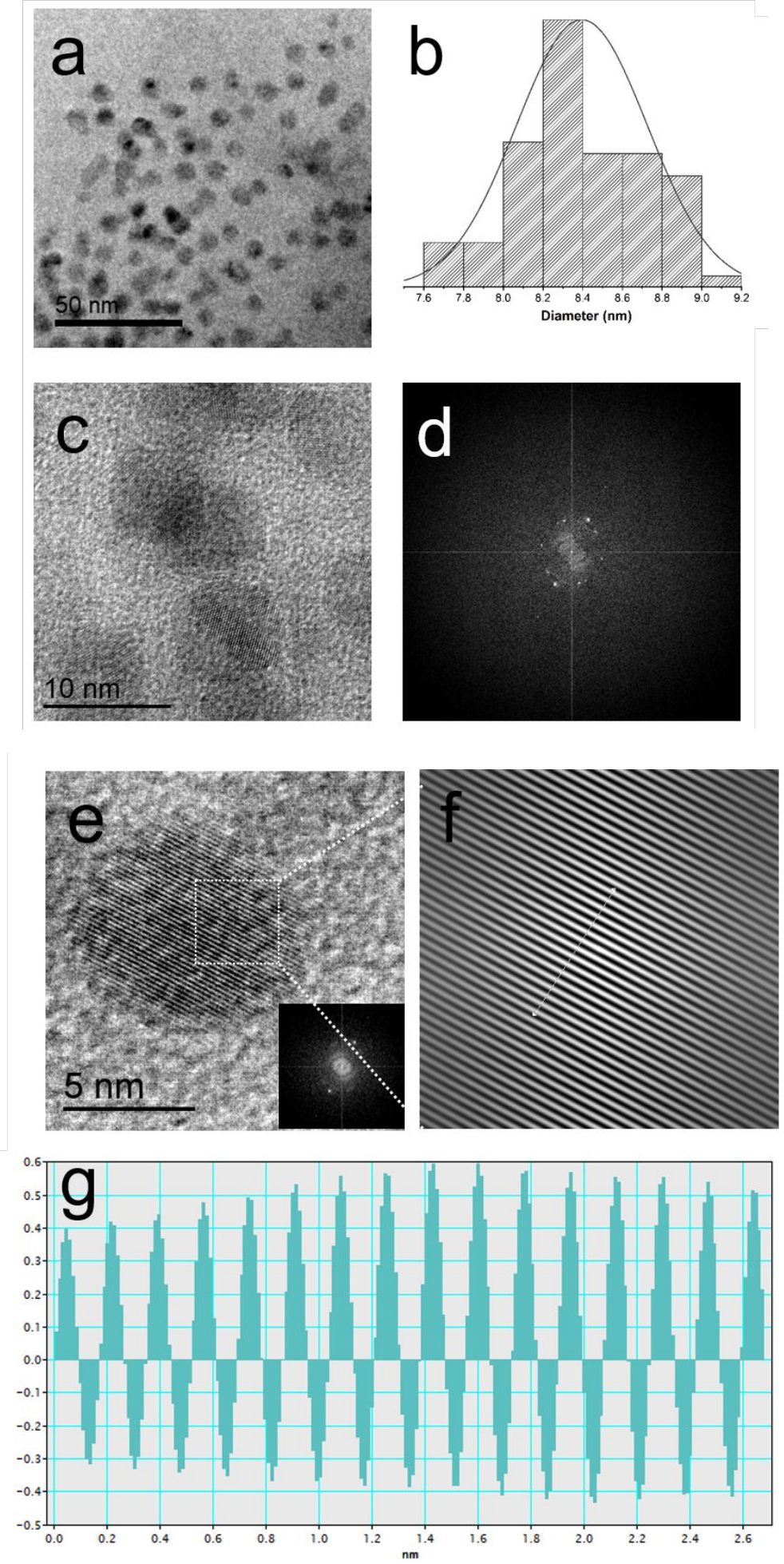

Figure s3. TEM image of red carbon quantum dots (a, c), Size distributions of the R-CDs (b). Fast Fourier transform (FFT) patterns of R-CDs (d), High resolution-TEM (HRTEM) images of R-CDs (e), Plane spacing of carbon quanta in high power transmission electron microscopy (f, g) 


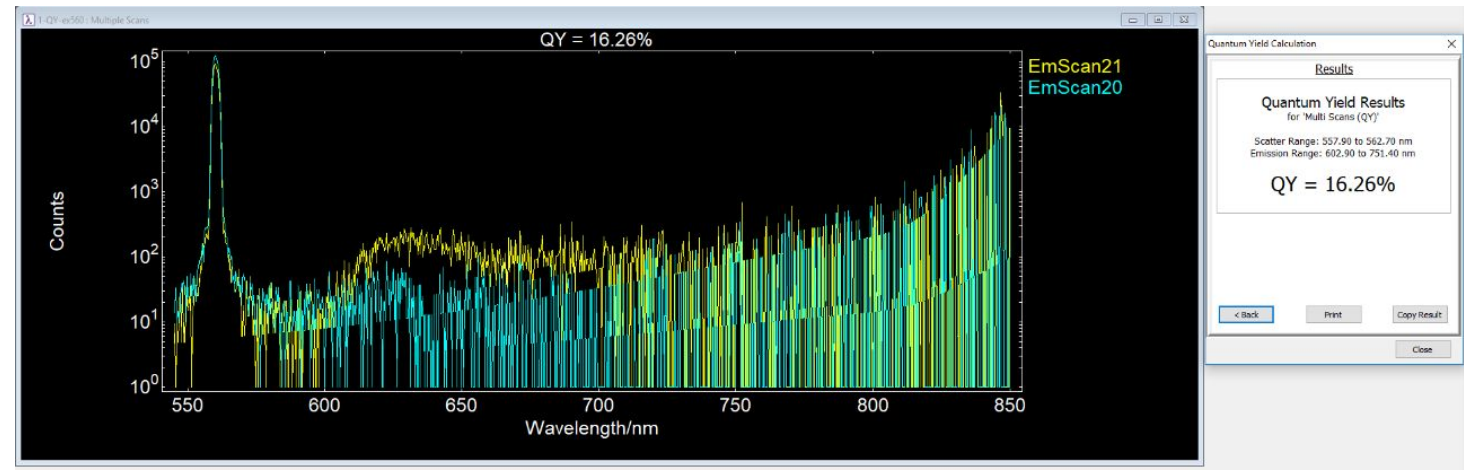

Figure s4. Absolute quantum yield (QY) of the R-CDs 


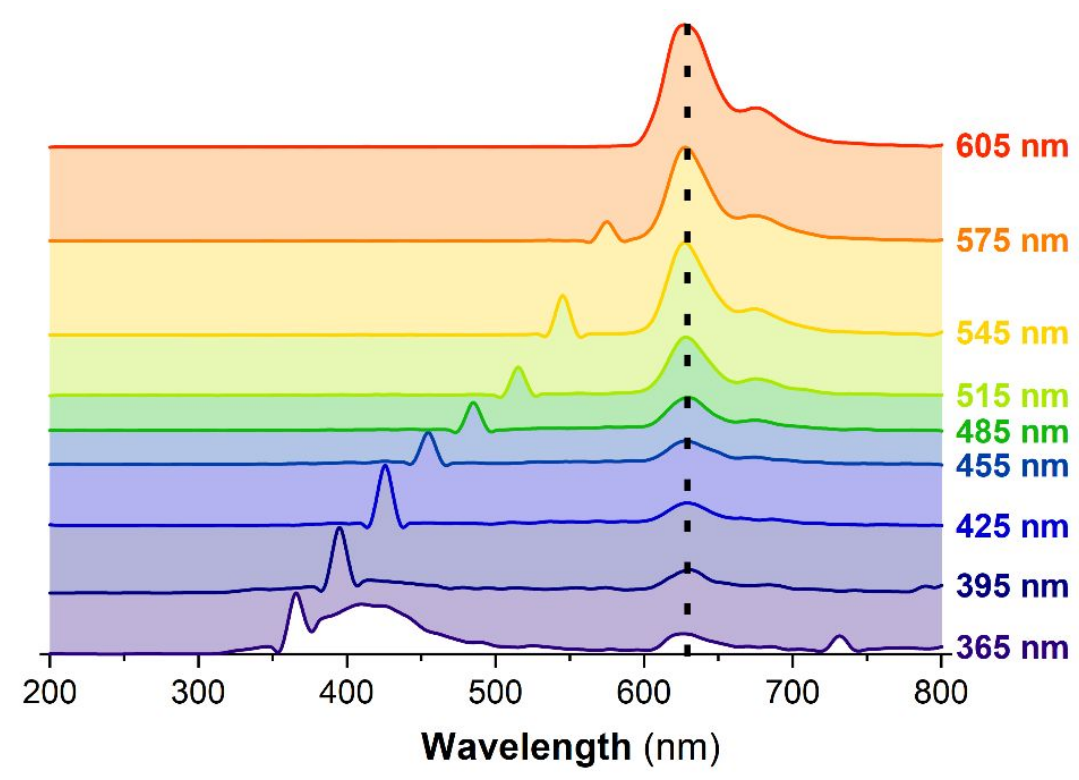

Figure s5: The excitation-dependent fluorescence emission of R-CDs ${ }^{1}$ 

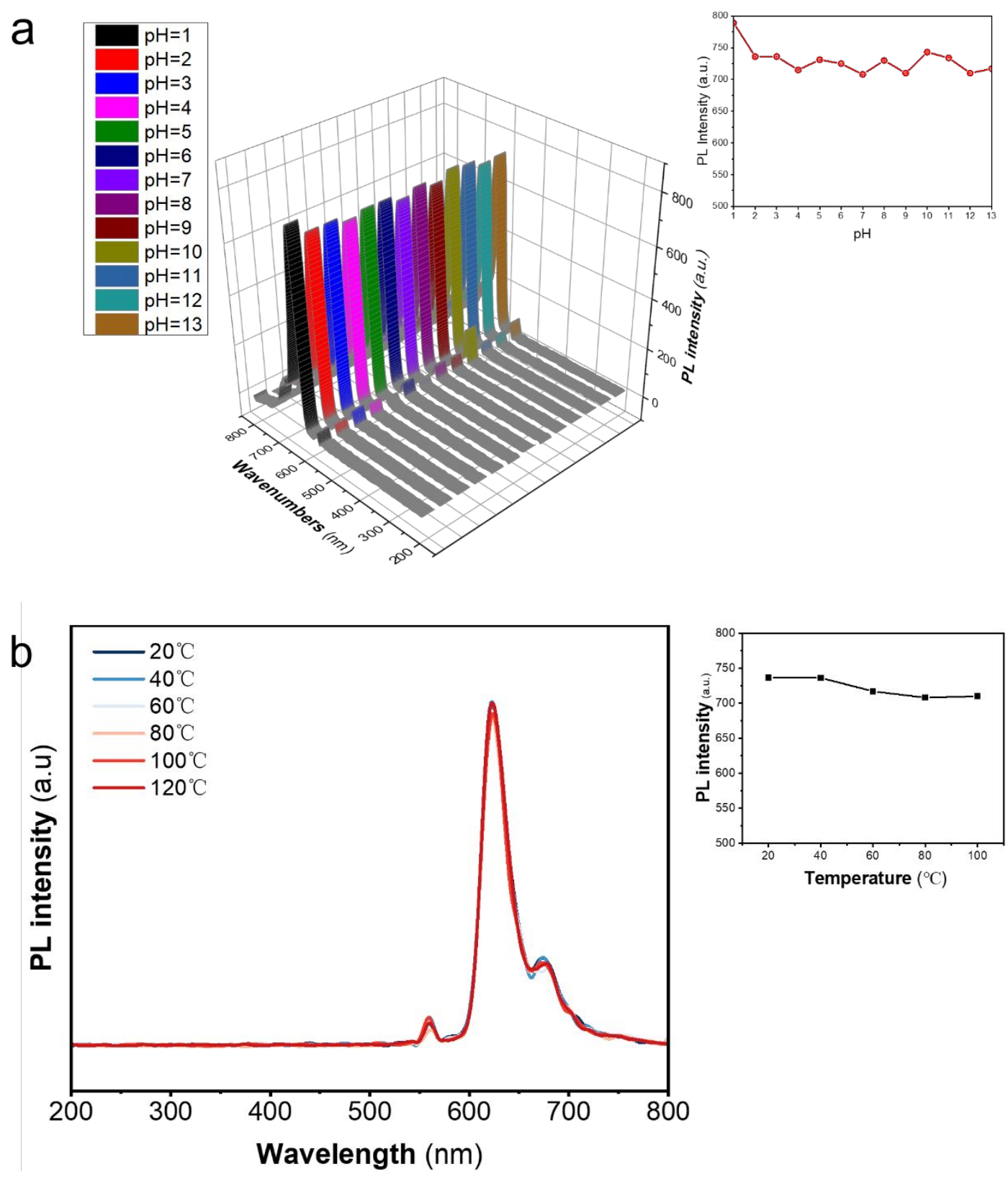

Figure s6. The $\mathrm{pH}$ (a) and temperature-dependent (b) PL spectra of R-CDs. 

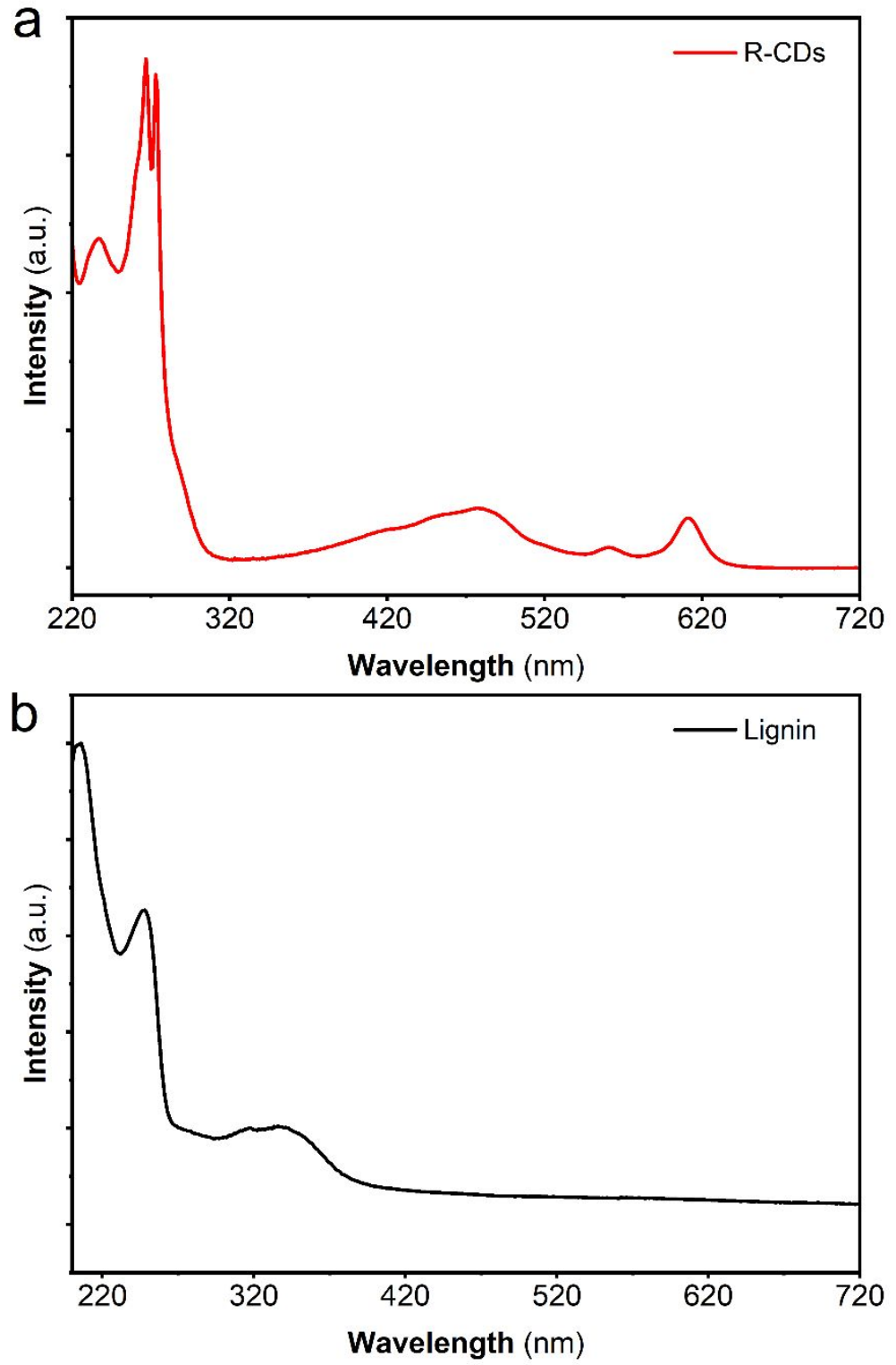

Figure s7. UV-vis absorption spectra of R-CDs (a) and lignin (b) 

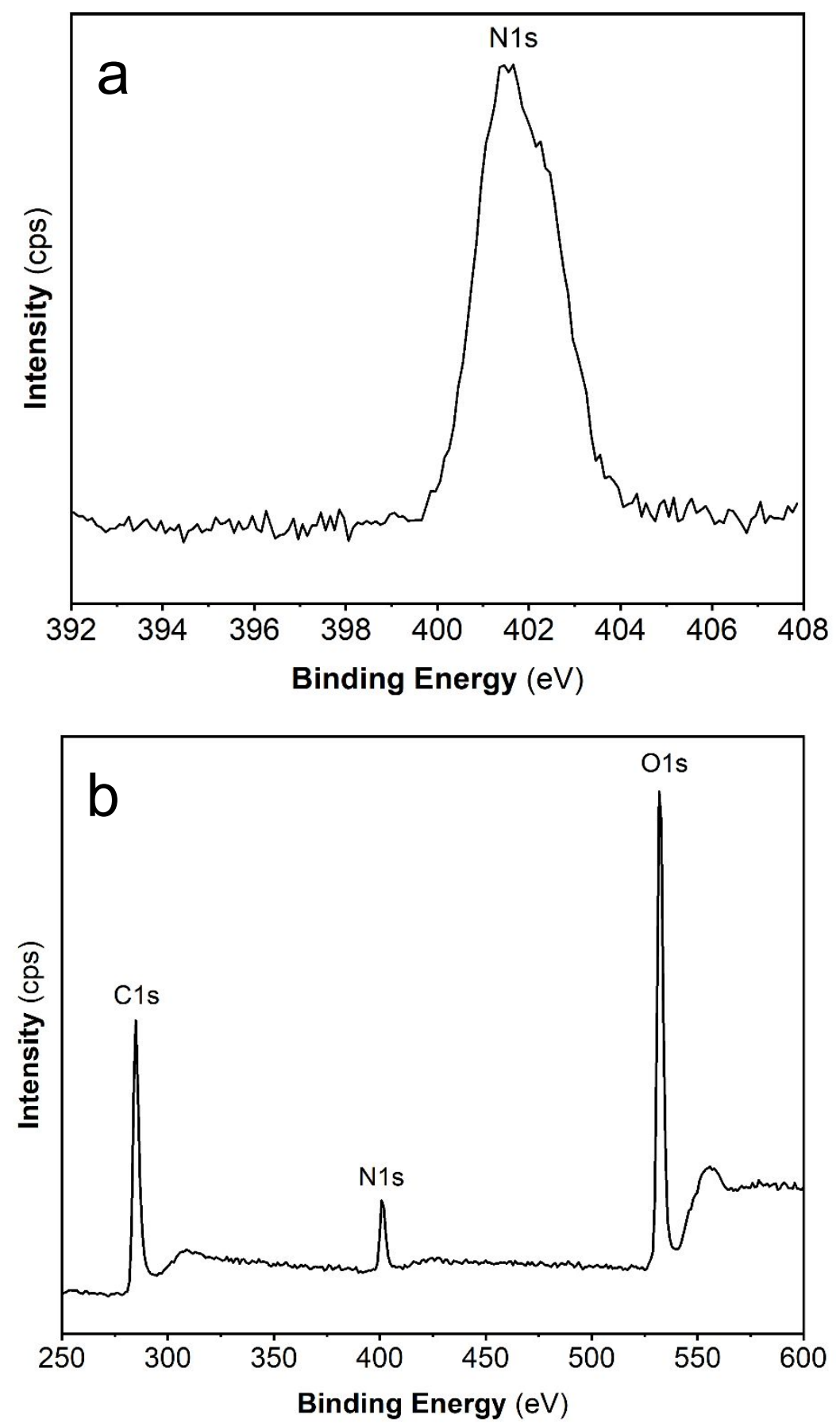

Figure s8. XPS wide spectra of R-CDs (a) and high-resolution XPS fitting results for N1s (b) 


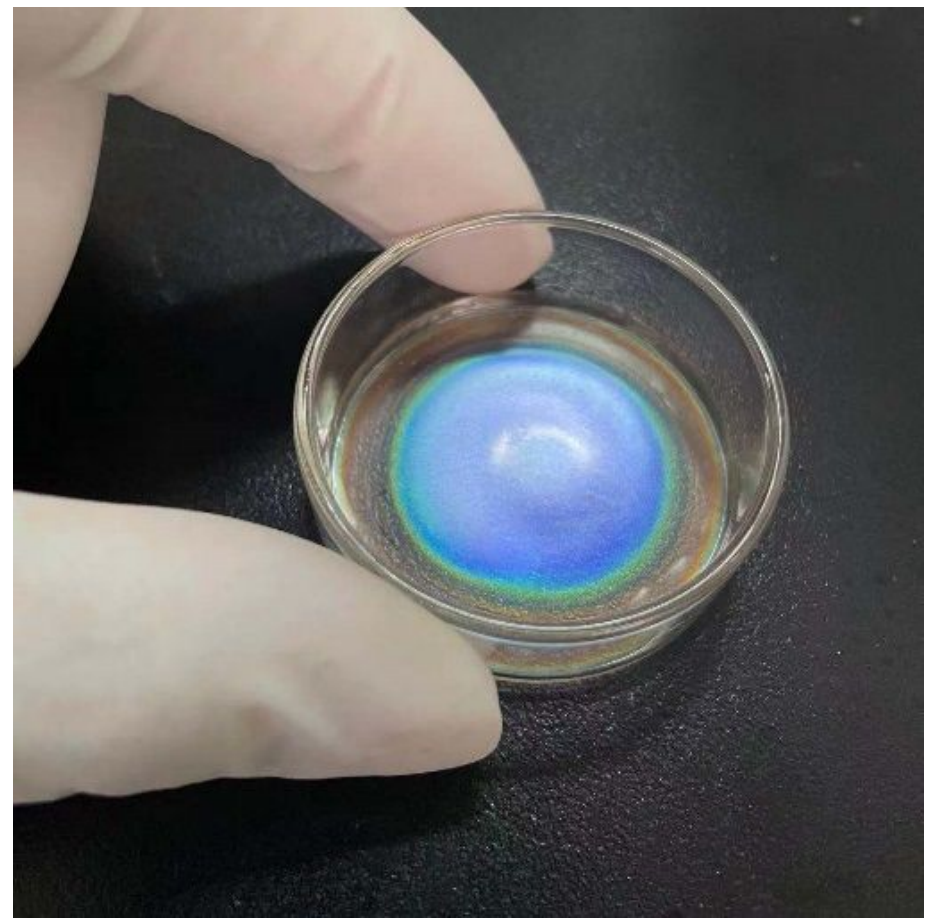

Figure s9. CNC film with rainbow effect 

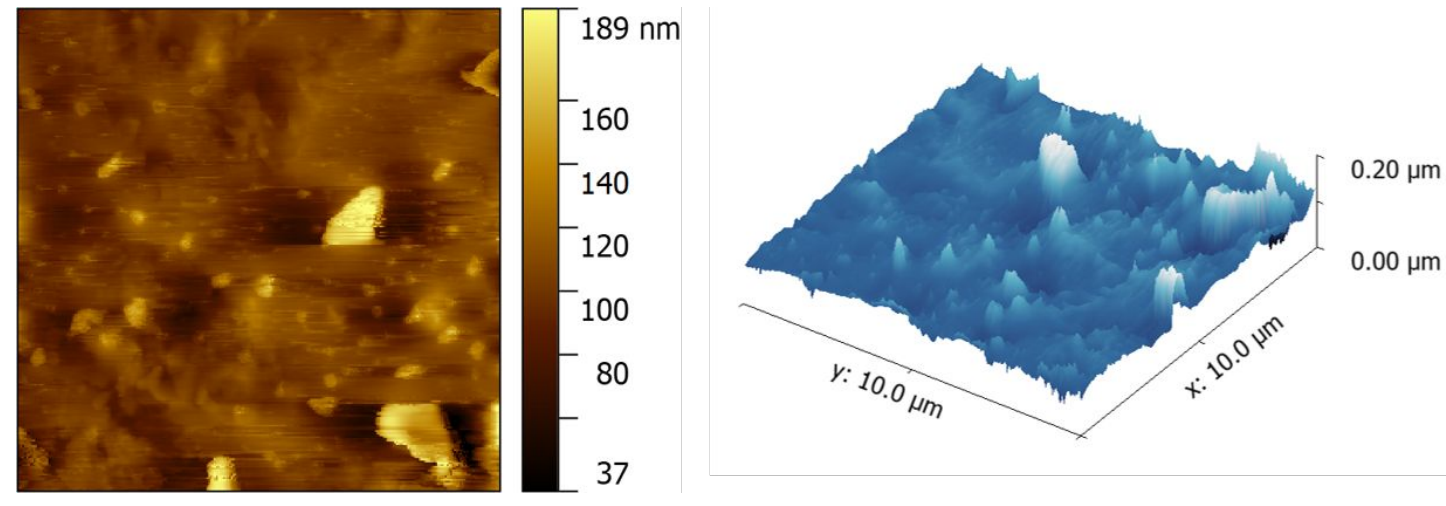

Figure s10. Atomic force microscope (AFM) image of CNC-CD-F 


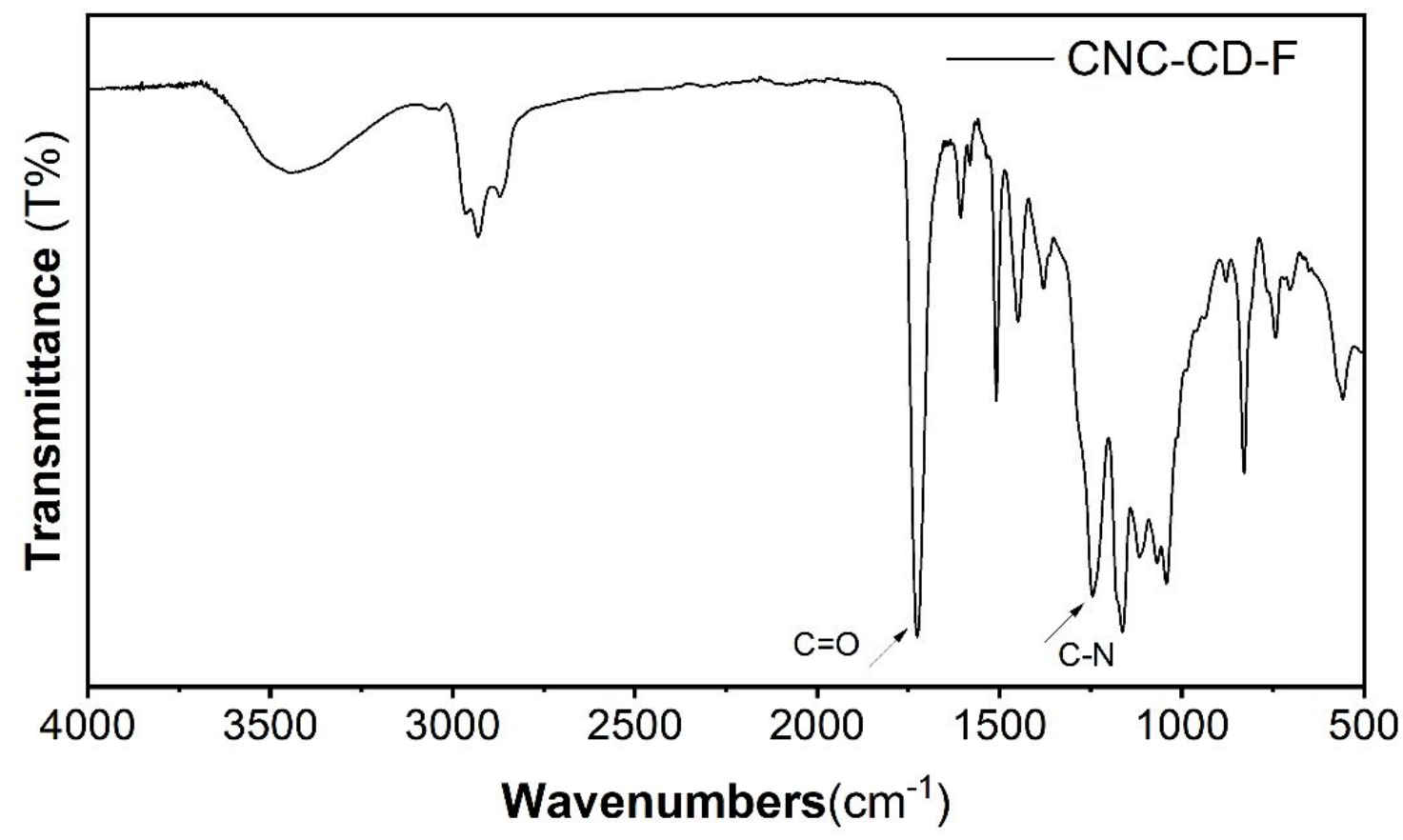

Figure s11. FTIR spectra of CNC-CD composite film 


\section{CNC-CD-F}

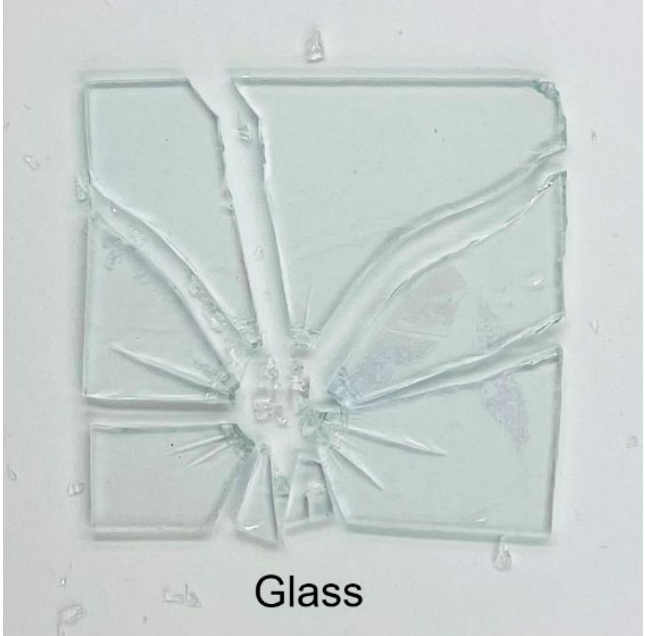

Figure s12. The impact resistance comparison of glass and CNC-CD-F 


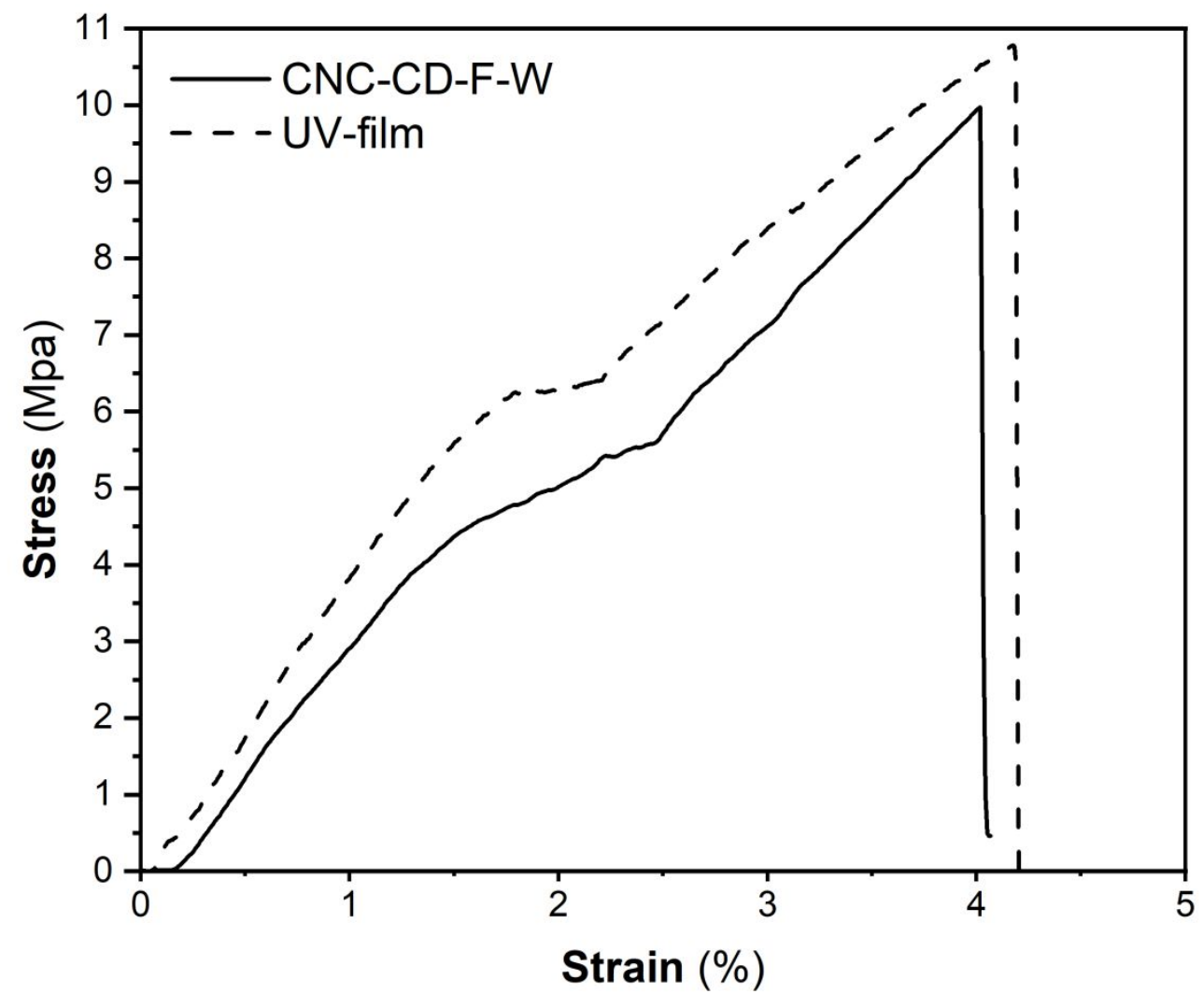

s13. The strain-stress spectra of CNC-CD-F after soaking in water 